\title{
Impact of inter- and intra-individual variation, sample storage and sampling fraction on human stool microbial community profiles
}

Yun Kit Yeoh ${ }^{1,2}$, Zigui Chen ${ }^{1,2}$, Mamie Hui ${ }^{1,2}$, Martin CS Wong ${ }^{2,3}$, Wendy CS Ho ${ }^{1}$, Miu Ling Chin ${ }^{1}$, Siew C Ng ${ }^{2,4,5}$, Francis KL Chan ${ }^{2,4}$, Paul KS Chan ${ }^{\text {corresp. } 1,2}$

1 Department of Microbiology, Faculty of Medicine, The Chinese University of Hong Kong, Shatin, Hong Kong SAR, China

3 Jockey Club School of Public Health and Primary Care, Faculty of Medicine, The Chinese University of Hong Kong, Shatin, Hong Kong SAR, China

4 Department of Medicine and Therapeutics, Faculty of Medicine, The Chinese University of Hong Kong, Shatin, Hong Kong SAR, China

5 State Key Laboratory of Digestive Disease, Li Ka Shing Institute of Health Sciences, The Chinese University of Hong Kong, Shatin, Hong Kong SAR, China

Corresponding Author: Paul KS Chan

Email address: paulkschan@cuhk.edu.hk

Stools are commonly used as proxies for studying human gut microbial communities as sample collection is straightforward, cheap and non-invasive. In large-scale human population surveys, however, sample integrity becomes an issue as it is not logistically feasible for researchers to personally collect stools from every participant. Instead, participants are usually given guidelines on sample packaging and storage, and asked to deliver their stools to a centralised facility. Here, we tested a number of delivery conditions (temperature, duration and addition of preservative medium) and assessed their effects on stool microbial community composition using 16S rRNA gene amplicon sequencing. The largest source of variability in stool community composition was attributable to interindividual differences regardless of delivery condition. Although the relative effect of delivery condition on community composition was small compared to inter-individual variability ( $1.6 \%$ vs. $60.5 \%$, permutational multivariate analysis of variance [PERMANOVA]) and temporal variation within subjects over 10 weeks (5.2\%), shifts in microbial taxa associated with delivery conditions were non-systematic and subject-specific. These findings indicated that it is not possible to model or accurately predict shifts in stool community composition associated with sampling logistics. Based on our findings, we recommend delivery of fresh, preservative-free stool samples to laboratories within $2 \mathrm{hr}$ either at ambient or chilled temperatures to minimise perturbations to microbial community composition. In addition, subsamples from different fractions of the same stool displayed a small (3.3\% vs. $72.6 \%$ inter-individual variation, PERMANOVA) but significant effect on community composition. Collection of larger sample volumes for homogenisation is recommended. 
1 Title: Impact of inter- and intra-individual variation, sample storage and 2 sampling fraction on human stool microbial community profiles

3 Yun Kit Yeoh ${ }^{1,2}$, Zigui Chen ${ }^{1,2}$, Mamie Hui ${ }^{1,2}$, Martin C. S. Wong ${ }^{2,3}$, Wendy C. S. Ho ${ }^{1}$, Miu Ling $4 \mathrm{Chin}^{1}$, Siew C. $\mathrm{Ng}^{2,4,5}$, Francis K. L. Chan ${ }^{2,4}$, Paul K. S. Chan ${ }^{1,2 *}$

$5 \quad{ }^{1}$ Department of Microbiology, Faculty of Medicine, The Chinese University of Hong Kong, Hong 6 Kong SAR, China.

$7 \quad{ }^{2}$ Centre for Gut Microbiota Research, Faculty of Medicine, The Chinese University of Hong Kong, 8 Hong Kong SAR, China.

9 3Jockey Club School of Public Health and Primary Care, Faculty of Medicine, The Chinese 10 University of Hong Kong, Hong Kong SAR, China.

$11{ }^{4}$ Department of Medicine and Therapeutics, Faculty of Medicine, The Chinese University of Hong 12 Kong, Hong Kong SAR, China.

$13{ }^{5}$ State Key Laboratory of Digestive Disease, Li Ka Shing Institute of Health Sciences, The Chinese 14 University of Hong Kong, Hong Kong SAR, China

15

$16 *$ Corresponding author

17 Email: paulkschan@cuhk.edu.hk 


\section{Abstract}

Stools are commonly used as proxies for studying human gut microbial communities as sample collection is straightforward, cheap and non-invasive. In large-scale human population surveys, however, sample integrity becomes an issue as it is not logistically feasible for researchers to personally collect stools from every participant. Instead, participants are usually given guidelines on sample packaging and storage, and asked to deliver their stools to a centralised facility. Here, we tested a number of delivery conditions (temperature, duration and addition of preservative medium) and assessed their effects on stool microbial community composition using 16S rRNA gene amplicon sequencing. The largest source of variability in stool community composition was attributable to inter-individual differences regardless of delivery condition. Although the relative effect of delivery condition on community composition was small compared to inter-individual variability ( $1.6 \%$ vs. $60.5 \%$, permutational multivariate analysis of variance [PERMANOVA] $)$ and temporal variation within subjects over 10 weeks $(5.2 \%)$, shifts in microbial taxa associated with delivery conditions were non-systematic and subject-specific. These findings indicated that it is not possible to model or accurately predict shifts in stool community composition associated with sampling logistics. Based on our findings, we recommend delivery of fresh, preservative-free stool samples to laboratories within $2 \mathrm{hr}$ either at ambient or chilled temperatures to minimise perturbations to microbial community composition. In addition, subsamples from different fractions of the same stool displayed a small $(3.3 \%$ vs. $72.6 \%$ inter-individual variation, PERMANOVA) but significant effect on community composition. Collection of larger sample volumes for homogenisation is recommended.

\section{Introduction}

The human gut microbiome has gained unprecedented appreciation for its many vital roles in health and disease. In recent years, studies have linked dysbiosis in gut microbial communities to conditions such as obesity (Turnbaugh et al., 2006), diabetes (Qin et al., 2012) and even neurodegenerative diseases such as Parkinson's (Bedarf et al., 2017) and Alzheimer's disease (Vogt et al., 2017), underscoring their roles in development, maturation and maintenance of health. Owing to this intimate association between gut microorganisms and their hosts, the gut microbiome has become an attractive target for healthcare practices that aim to restore and promote health by manipulating microbial communities of the gut. This undertaking requires an underlying knowledge of the "healthy" baseline composition and function of gut microbial communities, and how they respond to host factors and environmental perturbations. To that end, various studies have examined the influence of demographics, environment and lifestyle habits over ecology of gut microbial communities in cohorts from various biogeographical backgrounds (Zeevi et al., 2015; Zhernakova et al., 2016; Falony et al., 2016; Rothschild et al., 2018), and generally agree that environment is a stronger determinant of gut microbiota composition relative to host factors such as ancestry and genetics. 
56 Currently, there are no reference gut microbiota profiles for the South Asian population in Hong 57 Kong. Since environment is commonly the strongest predictor of gut microbiota profiles 58 (Rothschild et al., 2018), it is necessary to assess whether the gut microbiota of distinct populations

59

60

61

62

63

64

65

66

67

68

69

70

71

72

73

74

75

76

77

78

79

80

81

82 are comparable to previously surveyed cohorts and validate whether trends observed from international studies apply to a local population while considering differences in demographics and lifestyles. To address this lack of a local gut microbiome dataset, we are initiating a gut microbial community survey targeted at the general Hong Kong public. One important consideration is that gut microbiome surveys face a challenge of balancing sampling logistics and study power (Sze \& Schloss, 2016) where hundreds to thousands of stool samples are often needed to account for unique population niches and discern potential confounding factors. Because of the large number of participants a population gut microbiome survey would entail, we wanted to formulate simple instructions for participants to deliver stools to a centralised laboratory while also preserving sample integrity as much as possible during transit.

Here, we tested the impact of commonly used sample delivery conditions on stool microbial community profiles. Parameters evaluated included delivery temperature, duration and use of a preservative medium. The impact on microbial community composition was assessed by $16 \mathrm{~S}$ ribosomal RNA gene (16S) amplicon profiling, and results were interpreted to determine the relative contributions of the evaluated parameters towards compositional differences in community composition. In addition, shifts in the relative abundances of specific microbial taxa associated with the tested parameters were assessed to determine whether perturbations in community composition due to sampling logistics could be generalised across samples and predicted in future sampling efforts.

8

\section{Materials and methods}

\section{Ethics statement and consent}

This study has been approved by the Joint Chinese University of Hong Kong-New Territories East Cluster Clinical Research Ethics Committee (reference number 2016.707). Written consent was obtained from all participants prior to collecting stool samples.

\section{Sample collection}

Stool samples were collected from three subjects on nine separate occasions. Collection was scheduled in three batches- stools were collected thrice over a period up to seven days to study short-term temporal variation in stool microbial communities, and again three to four weeks and eight to ten weeks after the first collection to study medium-term temporal variability (Fig. 1). Subjects defecated on site and the whole stool was collected in a sterile container. The first stool collected from all three subjects at day 0 were aliquoted and assigned to four treatments intended 
92 to simulate how various delivery conditions could impact outcomes of $16 \mathrm{~S}-$ based gut microbial

93

94

95

96

97

98

99

100

101

102

103

104

105

106

107

108

109

110

111

112

113

114

115

116

117

118

119

120

121

122

123

124

125

126

127

128

community surveys: (i) frozen at $-80^{\circ} \mathrm{C}$ within 5 min of defecation, (ii) ambient temperature for 2 , 5 and 24 hours then frozen at $-80^{\circ} \mathrm{C}$, (iii) chilled with cool packs for 2, 5 and 24 hours in an insulated container then frozen at $-80{ }^{\circ} \mathrm{C}$, and (iv) stored in a stool nucleic acid collection and preservation medium (catalogue number 63700, Norgen Biotek Corp., Canada) and frozen at -80 ${ }^{\circ} \mathrm{C}$ immediately or after 2, 5 and 24 hours at ambient temperature. Subsequent stool samples collected from day 1 onwards were immediately frozen at $-80^{\circ} \mathrm{C}$.

\section{DNA extraction and 165 amplicon sequencing}

DNA was extracted in triplicate from $0.1 \mathrm{~g}$ homogenised fractions of stool using the QIAGEN DNeasy PowerSoil Kit following manufacturer's instructions. Following extraction, the V3 and V4 variable regions of the $16 \mathrm{~S}$ gene were amplified by polymerase chain reaction (PCR) in $25 \mu 1$ reactions with the following master mix recipe: $1 \mathrm{X}$ PCR buffer, $0.2 \mathrm{mM}$ of each $\mathrm{dNTP}, 1 \mathrm{mM}$ $\mathrm{MgCl}_{2}, 0.2 \mu \mathrm{M}$ of each forward (341F: CCTACGGGNGGCWGCAG) and reverse (806R: GGACTACNVGGGTWTCTAAT) primers (Klindworth et al., 2013; Apprill et al., 2015), 0.625 U Hot Start plus DNA Taq polymerase and molecular biology grade water. Thermocycler settings were as follows: $95{ }^{\circ} \mathrm{C}$ for $3 \mathrm{~min}$, followed by 15 cycles of $94{ }^{\circ} \mathrm{C}$ for $45 \mathrm{~s}, 55^{\circ} \mathrm{C}$ for $30 \mathrm{~s}$ and $72{ }^{\circ} \mathrm{C}$ for $3 \mathrm{~min}$, followed by 10 cycles of $94{ }^{\circ} \mathrm{C}$ for $45 \mathrm{~s}, 60^{\circ} \mathrm{C}$ for $30 \mathrm{~s}$ and $72{ }^{\circ} \mathrm{C}$ for $3 \mathrm{~min}$, and finally $72{ }^{\circ} \mathrm{C}$ for $10 \mathrm{~min}$. Four DNA-free negative controls using water were included in the PCR. Successful PCR amplification was verified by visualising products on an agarose gel. Sequencing adapters and multiplex indices were added to the PCR products in a second $25-\mu 1$ PCR reaction with the following master mix recipe: $1 \mathrm{X}$ PCR buffer, $0.2 \mathrm{mM}$ of each $\mathrm{dNTP}, 1 \mathrm{mM} \mathrm{MgCl}{ }_{2}, 0.2$ $\mathrm{mM}$ of each Nextera index 1 and 2 primers, $0.1 \mu \mathrm{M}$ of each forward and reverse Golay index, $0.625 \mathrm{U}$ Hot Start plus DNA Taq polymerase and molecular biology grade water. Thermocycler settings were as follows: $95^{\circ} \mathrm{C}$ for $3 \mathrm{~min}$, followed by 10 cycles of $94{ }^{\circ} \mathrm{C}$ for $45 \mathrm{~s}, 63{ }^{\circ} \mathrm{C}$ for $30 \mathrm{~s}$ and $72{ }^{\circ} \mathrm{C}$ for $3 \mathrm{~min}$, and finally $72{ }^{\circ} \mathrm{C}$ for $10 \mathrm{~min}$. Amplicons were purified using the QIAquick Gel Extraction Kit. Purified final products including the four negative controls were sent to the Genomics Resource Core Facility at Cornell University for 2x300 bp sequencing on an Illumina MiSeq using the v3 MiSeq Reagent Kit.

\section{S sequence data processing}

Demultiplexed raw sequence data were imported into QIIME 2 v2017.12 (https:/github.com/ qiime2 /qiime2). Using the DADA2 workflow (Callahan et al., 2016) in QIIME 2, primer and low quality sequences were trimmed, and remaining reads subsequently denoised and merged. Alpha diversity metrics were calculated based on the counts table produced by DADA2, normalised to 1086 sequences per sample (Fig. S1d). To assign taxonomy to the sequences, a classifier was first trained on sequences extracted from the SILVA 16S database release 128 (Quast et al., 2013) using 
129 the 16S gene V3-4 universal primer sequences. This classifier was then run on the representative 130 sequences produced by DADA2 to assign probable taxonomies to the corresponding sequences. 131 The final counts table based on exact sequence variants (ESVs) was exported from QIIME2, 132 chloroplast sequences removed, and used as input in $\mathrm{R}$ for statistical analysis.

133

134

135

136

137

138

139

140

141

142

143

144

145

146

147

148

149

150

151

152

153

154

155

156

157

158

159

160

161

162

\section{$16 S$ amplicon-based microbial community composition analyses}

The resulting ESV counts table from QIIME2 was imported together with sample metadata into R v3.4.1. A centered log-ratio transformation was applied to ESV counts before downstream analyses to ensure that the counts fulfilled assumptions of independence between predictor variables for statistical analyses (explained in Lê Cao et al., 2016). Permutational multivariate analysis of variance (PERMANOVA) was used to assess whether factors such as subjectspecificity, sampling time point and the simulated storage conditions significantly influenced community composition, as well as to determine the amount of variation in community composition attributable to each of these experimental factors. Principal component analysis (PCA) was used to visualise the clustering of samples based on their compositional similarities. Association of ESVs to experimental factors were tested using generalised linear models (GLMs) and sparse partial least squares discriminant analysis (SPLSDA). PERMANOVA, PCA and GLMs are implemented in the vegan R package v2.4-6 (Oksanen et al., 2016), and SPLSDA implemented in the mixOmics R package v6.3.1 (Rohart et al., 2017). Statistical power was assessed using the HMP R package (La Rosa et al., 2012). Figures were edited in Inkscape v0.92 for clarity. All data are provided as supplemental files 1 to 4 .

\section{Data Availability}

Raw sequence data generated for this study are available in the Sequence Read Archive under BioProject accession PRJNA450690.

\section{Results}

Effect of storage conditions relative to inter-individual differences and intra-individual temporal variation

PCR amplicons from a total of 189 stool samples (Table S1) were pair-end sequenced on an Illumina MiSeq platform producing 886,252 reads, with a median of 4,556 reads per sample. Reads were quality-filtered to remove adaptor, primer, low-quality and chloroplast sequences, producing a final count of 475,361 reads. Of four DNA-free negative controls included in the PCR and sequencing workflow, two contained zero reads while the other two had three and four reads each. 
163 These controls were excluded from downstream statistical analyses. A total of seven samples each

164

165

166

167

168

169

170

171

172

173

174

175

176

177

178

179

180

181

182

183

184

185

186

187

188

189

190

191

192

193

194

195

196

197

198

199

200

containing less than 1,000 reads were also excluded, leaving 182 samples.

To determine which parameters were most strongly associated with community composition, we first applied a centered log-ratio transformation on $16 \mathrm{~S}$ counts data and then performed a PCA. Samples clustered according to their respective subjects based on community composition regardless of storage conditions or sampling time point (Fig. 2d), indicating that inter-individual variation had an overarching influence when comparing stool microbial communities among multiple subjects. Subject-to-subject stool microbial community specificity was also partially reflected in alpha diversity metrics including observed species, Shannon and Faith's phylogenetic diversity indices, in which samples from one subject (subject B) were consistently more species rich and phylogenetically diverse compared to samples from the other two subjects across all nine sampling time points ( $<<0.001$, Kruskal-Wallis test) (Fig. S1). Next, we performed PCA on samples from each subject separately and observed secondary clustering by sampling time point and transit storage conditions in all three subjects (Fig. 2a-c). Stool communities collected across multiple days tended to cluster together relative to samples from weeks apart, reflecting shifts in community composition over short and medium-term durations (Fig. S2). This overall sequence of sample clustering beginning with between-subject variability, followed by within-subject temporal variability and sample storage conditions was supported by a permutational multivariate analysis of variance (PERMANOVA) indicating that subjects (accounting for $60.5 \%$ of the total variance in community composition), sampling time point $(5.2 \%)$ and storage conditions $(1.6 \%)$ were significantly associated with community composition in descending size of effect (Table 1). Storage duration $\left(2,5\right.$ or 24 hours) before transferring samples to $-80{ }^{\circ} \mathrm{C}$ were also significantly associated with shifts in community compositions in the three storage conditions (ambient, chilled and preservative) (PERMANOVA, Table 1).

\section{Effect of preservation medium on stool community composition}

To specifically investigate the effects of sample storage conditions during transport to the laboratory on community composition, we performed PCA on community composition of stools that were subjected to all three storage conditions (i.e. stools from the first sampling time point on day 0). Samples were analysed separately by subject to remove the large influence of interindividual variability. In all three subjects, PCA ordination of stool community composition revealed that samples stored in preservative medium often clustered to the exclusion of fresh frozen, ambient temperature or chilled samples (Fig. 3a-c). In comparison, community compositions of samples without preservation buffer were comparatively more similar to each other irrespective of storage condition (Fig. 3a-c). Interestingly, effects of the preservation medium on alpha diversity was only significant in one subject (subject B, Kruskal-Wallis tests, $p$ $<0.05$ ) (Fig. S3), indicating that the preservation medium tested here introduced inconsistent biases into the resident stool community across samples. To examine which ESVs were enriched in the 
201

202

203

204

205

206

207

208

209

210

211

212

213

214

215

216

217

218

219

220

221

222

223

224

225

226

227

228

229

230

231

232

233

234

235

236

237

238

239

preserved samples, we used a GLM to predict ESV associations with the use of preservative medium based on centered log-ratio transformed $16 \mathrm{~S}$ counts. Using data from all three subjects, the model predicted 51 ESVs significantly associated with preservation medium treatment (false discovery rate-adjusted $\mathrm{p}<0.05$ ), 44 of which were enriched in preserved samples (Table S2). These associations were supported by an SPLSDA implemented in the mixOmics R package, in which 37 ESVs were overlapping between the two methods (GLM and SPLSDA, Table S2). In total, 26 of the 37 ESVs were clostridial. When communities were analysed separately by subject, there was little overlap in the ESVs associated with preservation medium among subjects (Table S3) which was most likely due to the strong inter-individual variability in stool communities. Nevertheless, there was a strong representation of clostridial ESVs associated with the use of preservation medium in all three subjects (Table S3). These results suggest that the preservation medium tested here may be enriching for certain clostridial taxa in stools. Thus, its effects need to be further evaluated in other stool and non-stool samples to determine whether the observed enrichment is specific to this study. Since these findings indicated that community composition in samples treated with preservation medium was altered relative to preservative-free options, we revisited the initial analysis of all 182 samples and excluded those stored in preservation medium. When the PERMANOVA was restricted to non-preserved samples, sample storage (ambient vs. chilled temperatures) now accounted for a smaller but still significant amount of variability $(0.6 \%$ vs $1.6 \%$ when preserved samples were included), and duration of storage was no longer associated with community composition when analysing stools from multiple subjects combined (Table 2).

\section{Effect of storage duration on community composition}

To more specifically identify at which time points significant differences could be detected in the community composition of stools stored at ambient and chilled temperatures relative to fresh frozen samples, we separately compared profiles of samples from 2, 5, and $24 \mathrm{~h}$ to the $0 \mathrm{~h}$ reference using PERMANOVA. At $2 \mathrm{~h}$, stools from all three subjects did not differ in community composition from their respective fresh frozen fractions regardless of ambient or chilled storage. At 5 and 24 h, only stools from subject B showed significant alterations in both ambient and chilled conditions whereas stool profiles of the other two subject did not differ from their respective $0 \mathrm{~h}$ fractions (Table S4). Similar to the non-systematic effects of the tested preservation medium on community composition, these observations further indicate that storage conditions can produce varying outcomes on stool community composition among individuals. We then assessed changes in the centered log-ratio transformed counts of each ESV across the 0,2,5 and $24 \mathrm{~h}$ time points using GLMs to identify taxa enriched or depleted over time. A total of 86 ESVs were implicated, of which 74 were enriched, eight depleted, and four both enriched and depleted among the three subjects (Table S5). While the number of ESVs enriched or depleted could be influenced by the relative nature of microbial community data, their taxonomic identities were specific to subjects and included a range of common gut taxa (Table S5). These observations indicate that there is likely no generalizable pattern in how stool community composition from multiple individuals 
240

241

242

243

244

245

246

247

248

249

250

251

252

253

254

255

256

257

258

259

260

261

262

263

264

265

266

267

268

269

270

271

272

273

274

275

276

changes over a $24 \mathrm{~h}$ storage duration. Within subjects, many ESVs enriched or depleted over time in the ambient and chilled conditions overlapped, indicating that temperature may not radically influence enrichment/depletions at least in the first 24 hours. Taken together, these results show that responses in stool community composition to storage conditions is specific to individuals and may not be easily accounted for in downstream analyses. While these shifts were small compared to inter-individual variability detected in stool community compositions (Table 2), their potential impacts on gut communities should be considered when interpreting survey results.

\section{Effect of sampling different stool fractions on community composition}

For each of the three subjects, we produced replicate community profiles from three separate fractions of the whole stool (from day 0 ) taken at least $5 \mathrm{~cm}$ apart to assess variability in community composition attributable to heterogeneity in microbial niches within stools. Subsampling of different fractions was significantly associated with distinct community compositions in two of three subject ( $p<0.05$; PERMANOVA) (Table S6, Fig. S4). We then combined the samples from all three subjects and performed a PERMANOVA to assess the relative influences of interindividual variability and intra-sample heterogeneity on stool community composition. The PERMANOVA indicated that in the absence of other experimental variables, differences in community composition linked to sampling different fractions of the same stools were subtle but still significant despite the stronger influence of inter-individual variation $\left(72.6 \%\right.$ vs. $3.3 \% \mathrm{R}^{2}$, PERMANOVA) (Table S6). This finding indicates that distinct fractions of the same stool may not necessarily be true biological replicates, therefore collecting a larger sample for homogenisation before storage may be necessary. Taken together, the variables investigated in this study ranked according to their effect on stool microbial community composition are: interindividual variation $>$ intra-individual temporal variation $>$ use of preservative media $>$ storage temperature $>$ storage duration (up to 24 hours) before long term freezing.

\section{Discussion}

Large-scale population gut microbiota surveys often face a logistical issue of procuring hundreds of stools while maintaining sample integrity. Typically, the ideal laboratory procedure for storing stools is often immediate freezing at $-80^{\circ} \mathrm{C}$ following defecation (Carroll et al., 2012, Fouhy et al., 2015), however, this workflow is not feasible in surveys involving large numbers of participants from the general public. A major concern with collection of stool and other biological samples intended for microbial community surveys is the issue of prolonged exposure to ambient temperatures during transit, leading to shifts in community composition (Cardona et al., 2012; Carroll et al., 2012; Guo et al., 2016). Biological samples are usually chilled or mixed with preservative media to suppress opportunistic microorganisms from flourishing and dominating community profiles (Vandeputte et al., 2017), although this problem may be somewhat alleviated 
277 by setting a maximum allowed transit duration. A few studies have compared the use of 278 preservation buffers in maintaining microbial community profiles and generally recommend its use especially when samples cannot be immediately frozen due to logistical constraints (Menke et al., 2017; Flores et al., 2015; Voight et al., 2015). Our results, however, show that preservation media may not be necessary for human stool samples for short transit durations of up to 24 hours as the use of a medium was associated with larger shifts in microbial community composition compared to samples stored at ambient and lowered temperatures (Fig. 3). While there is a possibility that the three samples collected in this study contained less appreciable abundances of fast-growing microorganisms favoured at ambient temperatures, our findings echo recommendations relating to storage options and durations made by Vandeputte and colleagues, in which they rated chilled buffer-free samples for up to 24 hours as the best option other than immediate freezing (Table 1 in Vandeputte et al., 2017). Nevertheless, we only tested one preservative medium on stools from three subjects and there may be alternatives that produce community profiles more comparable to buffer-free storage conditions. Song and colleagues previously tested five sample preservative media on human stool samples and reported a general increase in Firmicutes taxa in samples preserved using ethanol (70\% and 95\%), RNALater or Whatman FTA cards but not OMNIgene Gut (Song et al., 2016). Our observations of increased relative abundances of clostridial ESVs (members of Firmicutes) in preserved samples (Table S2) was consistent with their findings. Although our data set presented here provided sufficient statistical power for discriminating preserved and non-preserved samples based on community profiles $(98.5 \%$ power at 0.05 alpha level, Monte-Carlo permutation of Dirichlet multinomial likelihood ratio implemented in HMP R package), additional tests inclusive of a larger number of subjects are required to determine whether composition and/or diversity of the resident stool microbial community influence performance of preservative media.

As highlighted above, our findings indicated that preservative media may not be necessary for stools collected and frozen within 2 hours (Fig. 3, Table S4). Stools stored at ambient and chilled temperatures produced community profiles more closely resembling those from fresh frozen samples, however, we noted that these outcomes were variable between subjects (Table S4). A few published studies have reported that storing stools at $4{ }^{\circ} \mathrm{C}$ for 24 hours did not significantly shift community profiles compared to fresh frozen $-80{ }^{\circ} \mathrm{C}$ controls (Bassis et al., 2017; Choo, Leong \& Rogers, 2015; Tedjo et al., 2015; Cardona at al., 2012). While profiles from two of our three subjects did not significantly change over 24 hours at ambient and chilled temperatures, significant shifts were detected in the stools of the third subject (subject B) at five hours post collection. Furthermore, when we attempted to identify taxa associated with these shifts, we observed that the relatively enriched and depleted ESVs were specific to subject and encompassed generic human gut taxa including various Bacteroidales and clostridial members (Table S5). In some instances, the same ESV present in multiple subjects showed enrichment in one but depletion or no change in relative abundance over time in other subjects. Community shifts over time in nonfrozen samples are often associated with reductions in community diversity as profiles become dominated by a limited number of taxa (Choo, Leong \& Rogers, 2015) due to factors such as 
317 altered temperatures relative to the normal human body or exposure to oxygen favouring aerobes 318 and/or facultative anaerobes (Chu et al., 2017). However, subject B's profiles were increased in 319 diversity (Fig. S3) due to depletion of a dominant Prevotella ESV from an average 26.0\% relative

320

321

322

323

324

325

326

327

328

329

330

331

332

333

334

335

336

337

338

339

340

341

342

343

344

345

346

347

348

349

350

351

352

353

354

355 abundance to $11.4 \%$ and $14.6 \%$ under ambient and chilled temperatures, respectively (Table S5). These results indicate that how stool communities respond to non-frozen storage varies by individual due to their specific configurations in stool microbial communities, and generalisations as to how stool community profiles respond over time after collection have to be made with caution. As Prevotella constitutes a major component of human gut communities and is a common feature in a large proportion of the human population (enterotype 2 described in Arumugam et al., 2011), changes as seen in subject B's profiles could severely impact the outcome of human population-based microbial community surveys.

The various human stool microbial community profiling studies mentioned here, like most other gut microbiome studies, involve homogenising samples to potentially reduce variability associated with subsampling (Vandeputte et al., 2017; Sinha et al., 2016; Choo, Leong \& Rogers, 2015; Tedjo et al., 2015; Carroll et al., 2012). The issue with subsampling small portions of a stool for DNA isolation is that microenvironments can harbour specific taxa as shown by a quantitative PCR study measuring abundances of common human gut taxa in non-homogenised stools (Gorzelak et al., 2015). Using 16S-based community profiling, we also detected significant differences in overall community composition in two of three subjects when subsamples derived from different fractions of the same stool were compared. Therefore, we recommend based on these observations collection of a larger stool sample and inclusion of a homogenisation step before samples are stored frozen. That said, differences in community composition linked to within-sample variability is relatively minor compared to other experimental factors such as inter-individual and temporal variation (Voight et al., 2015) and is unlikely to influence interpretation of a multi-subject stool community survey.

The biggest limitation in this study is the limited number of subjects involved as we subsequently showed that inter-individual variability largely influences how profiles respond to the storage conditions tested. Future surveys should include a larger population of subjects to capture greater variability in stool community composition, which may help discern general patterns in how stool taxa respond to storage conditions. Another issue not addressed here is the influence of oxygen on stool community composition. One study demonstrated that exposure to oxygen primarily lowered relative abundances of Faecalibacterium and Megamonas, while increasing that of Bacteroides (Chu et al., 2017). These alterations could be minimised by storing samples in air-tight containers with oxygen scavengers, but we have not incorporated such elements in our sample collection workflow as it introduces additional inconveniences to participants of stool community surveys during self-sampling. Furthermore, Chu and colleagues showed that using standard 16S sequencing, community profiles from stools processed under anaerobic and aerobic conditions equally varied from control samples (anaerobic with cysteine as reducing agent to remove oxygen from solution) (fig. 1A in Chu et al, 2017). Lastly, when comparing the findings presented here 
356

357

358

359

360

361

362

363

364

365

366

367

368

369

370

371

372

373

374

375

376

377

378

379

380

381

382

383

384

385

386

387

388

389

with other studies attention should be paid to the primers used as primer choice is widely known to influence 16S-based community compositional profiles (Walker et al., 2015; Engelbrektson et al., 2010).

\section{Conclusion}

Stool sample delivery conditions and subsampling biases are known to alter microbial community composition. The use of preservative media, while meant to lessen such alterations, resulted in non-systematic, non-taxa specific but subject-specific changes in stool community profiles. However, when compared to inter-individual differences and community variations within an individual over time, effects due to delivery conditions and biases in sample fractions are small and unlikely to obscure inter-individual variability in surveys involving multiple subjects. Nevertheless, the effects of sample collection strategies should be given consideration especially when dealing with sensitive applications such as faecal microbiota transplants, which are reliant on exact resident microbial species.

\section{Acknowledgement}

We wish to thank the stool donors for participating in this study, and reviewers for providing excellent feedback and suggestions.

\section{References}

Apprill A, McNally S, Parsons R, Weber L. 2015. Minor revision to V4 region SSU rRNA 806R gene primer greatly increases detection of SAR11 bacterioplankton. Aquatic Microbial Ecology 75:129-137.

Arumugam M, Raes J, Pelletier E, Le Paslier D, Yamada T, Mende DR, Fernandes GR, Tap J, Bruls T, Batto JM, Bertalan M, Borruel N, Casellas F, Fernandez L, Gautier L, Hansen T, Hattori M, Hayashi T, Kleerebezem M, Kurokawa K, Leclerc M, Levenez F, Manichanh C, Nielsen HB, Nielsen T, Pons N, Poulain J, Qin J, Sicheritz-Ponten T, Tims S, Torrents D, Ugarte E, Zoetendal EG, Wang J, Guarner F, Pedersen O, de Vos WM, Brunak S, Doré J; MetaHIT Consortium, Antolín M, Artiguenave F, Blottiere HM, Almeida M, Brechot C, Cara C, Chervaux C, Cultrone A, Delorme C, Denariaz G, Dervyn R, Foerstner KU, Friss C, van de Guchte M, Guedon E, Haimet F, Huber W, van Hylckama-Vlieg J, Jamet A, Juste C, Kaci G, Knol J, Lakhdari O, Layec S, Le Roux K, Maguin E, Mérieux A, Melo Minardi R, M'rini C, Muller J, Oozeer R, Parkhill J, Renault P, Rescigno M, Sanchez N, Sunagawa S, Torrejon A, Turner K, Vandemeulebrouck G, Varela E, Winogradsky Y, Zeller G, Weissenbach J, Ehrlich 

10.1038/nature09944.

Bassis CM, Moore NM, Lolans K, Seekatz AM, Weinstein RA, Young VB, Hayden MK, CDC Prevention Epicenters Program. 2017. Comparison of stool versus rectal swab samples and storage conditions on bacterial community profiles. BMC Microbiology 17:78. DOI: 10.1186/s12866-017-0983-9.

Bedarf JR, Hildebrand F, Coelho LP, Sunagawa S, Bahram M, Goeser F, Bork P, Wüllner U. 2017. Functional implications of microbial and viral gut metagenome changes in early stage L-DOPA-naïve Parkinson's disease patients. Genome Medicine 9:39. DOI: 10.1186/s13073017-0428-y.

401

Callahan BJ, McMurdie PJ, Rosen MJ, Han AW, Johnson AJA, Holmes SP. 2016. DADA2:

402 High-resolution sample inference from Illumina amplicon data. Nature Methods 13:581.

403 Cardona S, Eck A, Cassellas M, Gallart M, Alastrue C, Dore J, Azpiroz F, Roca J, Guarner F, 404 Manichanh C. 2012. Storage conditions of intestinal microbiota matter in metagenomic analysis. BMC Microbiology 12:158. DOI: 10.1186/1471-2180-12-158.

405

406

Carroll IM, Ringel-Kulka T, Siddle JP, Klaenhammer TR, Ringel Y. 2012. Characterization of

407 the Fecal Microbiota Using High-Throughput Sequencing Reveals a Stable Microbial Community during Storage. PLOS ONE 7:e46953. DOI: 10.1371/journal.pone.0046953.

Choo JM, Leong LE, Rogers GB. 2015. Sample storage conditions significantly influence faecal microbiome profiles. Scientific Reports 5:16350. DOI: 10.1038/srep16350. preparation of fecal microbiota transplantations. PLOS ONE 12: e0170922. DOI: 10.1371/journal.pone.0170922. P. 2010. Experimental factors affecting PCR-based estimates of microbial species richness and evenness. ISME Journal 4. DOI: 10.1038/ismej.2009.153. Valles-Colomer M, Vandeputte D, Tito RY, Chaffron S, Rymenans L, Verspecht C, De Sutter L, Fu J, Henckaerts L, Zhernakova A, Wijmenga C, Raes J. 2016. Population-level analysis of gut microbiome variation. Science 352:560-564. DOI: 10.1126/science.aad3503. freezing effects on microbial composition of human stool. Microbiome 3:33. DOI: 10.1186/s40168-015-0092-7. 
424

425

426

427

428

429

430

431

432

433

434

435

436

437

438

439

440

441

442

443

444

445

446

447

448

449

450

451

452

453

454

455

456

Fouhy F, Deane J, Rea MC, O’Sullivan Ó, Ross RP, O’Callaghan G, Plant BJ, Stanton C. 2015. The Effects of Freezing on Faecal Microbiota as Determined Using MiSeq Sequencing and Culture-Based Investigations. PLOS ONE 10:e0119355. DOI: 10.1371/journal.pone.0119355.

Gorzelak MA, Gill SK, Tasnim N, Ahmadi-Vand Z, Jay M, Gibson DL. 2015. Methods for Improving Human Gut Microbiome Data by Reducing Variability through Sample Processing and Storage of Stool. PLOS ONE 10:e0134802. DOI: 10.1371/journal.pone.0134802.

Guo Y, Li SH, Kuang YS, He JR, Lu JH, Luo BJ, Jiang FJ, Liu YZ, Papasian CJ, Xia HM, Deng HW, Qiu X. 2016. Effect of short-term room temperature storage on the microbial community in infant fecal samples. Scientific Reports 6:26648.

Klindworth, A, Pruesse E, Schweer T, Peplies J, Quast C, Horn M, Glöckner FO. 2013. Evaluation of general 16S ribosomal RNA gene PCR primers for classical and next-generation sequencing-based diversity studies. Nucleic Acids Research 41:e1. DOI: 10.1093/nar/gks808

La Rosa PS, Brooks JP, Deych E, Boone EL, Edwards DJ, Wang Q, Sodergren E, Weinstock G, Shannon WD. 2012. Hypothesis Testing and Power Calculations for Taxonomic-Based Human Microbiome Data. PLOS ONE 7:e52078. DOI: 10.1371/journal.pone.0052078.

Lê Cao KA, Costello M-E, Lakis VA, Bartolo F, Chua XY, Brazeilles R, Rondeau P. 2016. MixMC: A Multivariate Statistical Framework to Gain Insight into Microbial Communities. PLOS ONE 11:e0160169. DOI: 10.1371/journal.pone.0160169.

Menke S, Gillingham MAF, Wilhelm K, Sommer S. 2017. Home-Made Cost Effective Preservation Buffer Is a Better Alternative to Commercial Preservation Methods for Microbiome Research. Frontiers in Microbiology 8:102. DOI: 10.3389/fmicb.2017.00102.

Oksanen J, Blanchet FG, Kindt R, Legendre P, Minchin PR, O’hara R, Simpson GL, Solymos P, Stevens MHH, Wagner H. 2013. Package 'vegan.' Community ecology package, version 2.

Qin J, Li Y, Cai Z, Li S, Zhu J, Zhang F, Liang S, Zhang W, Guan Y, Shen D, Peng Y, Zhang D, Jie Z, Wu W, Qin Y, Xue W, Li J, Han L, Lu D, Wu P, Dai Y, Sun X, Li Z, Tang A, Zhong S, Li X, Chen W, Xu R, Wang M, Feng Q, Gong M, Yu J, Zhang Y, Zhang M, Hansen T, Sanchez G, Raes J, Falony G, Okuda S, Almeida M, LeChatelier E, Renault P, Pons N, Batto J-M, Zhang Z, Chen H, Yang R, Zheng W, Li S, Yang H, Wang J, Ehrlich SD, Nielsen R, Pedersen O, Kristiansen K, Wang J. 2012. A metagenome-wide association study of gut microbiota in type 2 diabetes. Nature 490:55-60. DOI: 10.1038/nature11450.

Quast C, Pruesse E, Yilmaz P, Gerken J, Schweer T, Yarza P, Peplies J, Glöckner FO. 2013. The SILVA ribosomal RNA gene database project: improved data processing and web-based tools. Nucleic Acids Research 41:D590-D596. DOI: 10.1093/nar/gks1219. 
457

458

459

460

461

462

463

464

465

466

467

468

469

470

471

472

473

474

475

476

477

478

479

480

481

482

483

484

485

486

487

488

489

490

491

Rohart F, Gautier B, Singh A, Lê Cao KA. 2017. mixOmics: An R package for 'omics feature selection and multiple data integration. PLOS Computational Biology 13:e1005752. DOI: 10.1371/journal.pcbi.1005752.

Rothschild D, Weissbrod O, Barkan E, Kurilshikov A, Korem T, Zeevi D, Costea PI, Godneva A, Kalka IN, Bar N, Shilo S, Lador D, Vila AV, Zmora N, Pevsner-Fischer M, Israeli D, Kosower N, Malka G, Wolf BC, Avnit-Sagi T, Lotan-Pompan M, Weinberger A, Halpern Z, Carmi S, Fu J, Wijmenga C, Zhernakova A, Elinav E, Segal E. 2018. Environment dominates over host genetics in shaping human gut microbiota. Nature 555:210-215.

Sinha R, Chen J, Amir A, Vogtmann E, Shi J, Inman KS, Flores R, Sampson J, Knight R, Chia N. 2016. Collecting Fecal Samples for Microbiome Analyses in Epidemiology Studies. Cancer epidemiology, biomarkers \& prevention 25:407-416. DOI: 10.1158/1055-9965.EPI-15-0951.

Song SJ, Amir A, Metcalf JL, Amato KR, Xu ZZ, Humphrey G, Knight R. 2016. Preservation methods differ in fecal microbiome stability, affecting suitability for field studies. mSystems $\mathbf{1}$, e00021-16. DOI: 10.1128/mSystems.00021-16.

Sze MA, Schloss PD. 2016. Looking for a Signal in the Noise: Revisiting Obesity and the Microbiome. mBio 7:e01018-16. DOI: 10.1128/mBio.01018-16.

Tedjo DI, Jonkers DMAE, Savelkoul PH, Masclee AA, van Best N, Pierik MJ, Penders J. 2015. The Effect of Sampling and Storage on the Fecal Microbiota Composition in Healthy and Diseased Subjects. PLoS ONE 10:e0126685. DOI: 10.1371/journal.pone.0126685.

Turnbaugh PJ, Ley RE, Mahowald MA, Magrini V, Mardis ER, Gordon JI. 2006. An obesityassociated gut microbiome with increased capacity for energy harvest. Nature 444:1027-131. DOI: $10.1038 /$ nature05414.

Vandeputte D, Tito RY, Vanleeuwen R, Falony G, Raes J. 2017. Practical considerations for large-scale gut microbiome studies. FEMS Microbiology Reviews 41:S154-S167. DOI: 10.1093/femsre/fux027.

Vogt NM, Kerby RL, Dill-McFarland KA, Harding SJ, Merluzzi AP, Johnson SC, Carlsson CM, Asthana S, Zetterberg H, Blennow K, Bendlin BB, Rey FE. 2017. Gut microbiome alterations in Alzheimer's disease. Scientific Reports 7:13537. DOI: 10.1038/s41598-01713601-y.

Voigt AY, Costea PI, Kultima JR, Li SS, Zeller G, Sunagawa S, Bork P. 2015. Temporal and technical variability of human gut metagenomes. Genome Biology 16:73. DOI: 10.1186/s13059-015-0639-8.

Walker AW, Martin JC, Scott P, Parkhill J, Flint HJ, Scott KP. 2015. 16S rRNA gene-based profiling of the human infant gut microbiota is strongly influenced by sample processing and PCR primer choice. Microbiome 3:26. DOI: 10.1186/s40168-015-0087-4. 
492 Zeevi D, Korem T, Zmora N, Israeli D, Rothschild D, Weinberger A, Ben-Yacov O, Lador D, 493 Avnit-Sagi T, Lotan-Pompan M, Suez J, Mahdi JA, Matot E, Malka G, Kosower N, Rein M, 494 Zilberman-Schapira G, Dohnalová L, Pevsner-Fischer M, Bikovsky R, Halpern Z, Elinav E, 495 Segal E. 2015. Personalized Nutrition by Prediction of Glycemic Responses. Cell 163:1079496 1094. DOI: 10.1016/j.cell.2015.11.001.

497 Zhernakova A, Kurilshikov A, Bonder MJ, Tigchelaar EF, Schirmer M, Vatanen T, Mujagic 498 Z, Vila AV, Falony G, Vieira-Silva S, Wang J, Imhann F, Brandsma E, Jankipersadsing SA, 499 Joossens M, Cenit MC, Deelen P, Swertz MA, Weersma RK, Feskens EJM, Netea MG, Gevers 500 D, Jonkers D, Franke L, Aulchenko YS, Huttenhower C, Raes J, Hofker MH, Xavier RJ, 501 Wijmenga C, Fu J. 2016. Population-based metagenomics analysis reveals markers for gut 502 microbiome composition and diversity. Science 352:565-569. DOI: 10.1126/science.aad3369. 
504 Figure Legends

505 Fig. 1. Overview of the stool microbial community survey and main experimental factors 506 investigated (categorized into four treatments). Three subjects were recruited to provide stools 507 three times over the span of three to four days (with the exception of subject B at day 0,1 and 7) 508 thrice over the duration of 72 days. Stools collected at day 0 were subjected to all four treatments 509 to assess their influence on microbial community composition. Community profiles from stools 510 immediately frozen at $-80^{\circ} \mathrm{C}$ were used as references for comparison. Subsequent stools collected 511 from day 1 onwards (sampling time points indicated in figure) were immediately frozen at $-80{ }^{\circ} \mathrm{C}$ 512 (only treatment 1).

513 Fig. 2. Principal component ordination of stool microbial community composition collected from 514 three subjects over 72 days. Community composition was determined by sequencing 16S rRNA 515 gene amplicons. Ordination of: A. Samples from subject A; B. Samples from subject B; C. 516 Samples from subject C; D. Samples from all three subjects. Each circle represents one sample, 517 and colour of circle represents storage condition: white, immediately frozen at $-80{ }^{\circ} \mathrm{C}$; red, ambient 518 temperature; blue, chilled; green, preservative medium at ambient temperature. Numbers in panels 519 A-C represent number of days subsequent samples were collected following initial sampling at 520 day 0 (day 0 samples immediately frozen are represented by the white circles). All samples 521 collected after day 0 were immediately frozen at $-80^{\circ} \mathrm{C}$.

522 Fig. 3. Principal component ordination of stool microbial community composition by storage 523 condition and duration. Community composition was determined by sequencing 16S rRNA gene 524 amplicons. A. Samples from subject A; B. Samples from subject B; C. Samples from subject C. 525 Each circle represents one sample. Colour of circle represents storage condition: white, 526 immediately frozen at $-80{ }^{\circ} \mathrm{C}$; red, ambient temperature; blue, chilled; green, preservative medium 527 at ambient temperature. Size of circle represents duration samples were subject to their respective 528 conditions before frozen long-term at $-80{ }^{\circ} \mathrm{C}$. 
Table $\mathbf{1}$ (on next page)

PERMANOVA of all samples. 
1 Table 1. PERMANOVA of all samples.

\begin{tabular}{rccccc} 
& $\begin{array}{c}\text { degrees of } \\
\text { freedom }\end{array}$ & Sums of squares & $\begin{array}{c}\mathrm{F} \\
\text { model }\end{array}$ & $\mathrm{R}^{2}$ & P value \\
\hline subject & 2 & 58888 & 198.1 & 0.605 & $<0.001$ \\
sampling time point & 8 & 5088 & 4.3 & 0.052 & $<0.001$ \\
storage temperature & 3 & 1597 & 3.6 & 0.016 & $<0.001$ \\
subject:sampling time point & 16 & 9071 & 3.8 & 0.093 & $<0.001$ \\
storage temperature:duration & 7 & 1184 & 1.1 & 0.012 & 0.04 \\
Residuals & 145 & 21555 & & 0.221 &
\end{tabular}

2

subject: three subjects $\mathrm{A}, \mathrm{B}$ and $\mathrm{C}$

sampling time point: days stools were collected

storage temperature before long-term freezing: immediately frozen, ambient, chilled, preservative at ambient duration of storage before long-term freezing: $0,2,5$ or $24 \mathrm{~h}$

7 
Table 2 (on next page)

PERMANOVA excluding preserved samples. 
1 Table 2. PERMANOVA excluding preserved samples.

\begin{tabular}{rccccc} 
& $\begin{array}{c}\text { degrees of } \\
\text { freedom }\end{array}$ & Sums of squares & $\begin{array}{c}\mathrm{F} \\
\text { model }\end{array}$ & $\mathrm{R}^{2}$ & P value \\
\hline subject & 2 & 45861 & 170.1 & 0.604 & $<0.001$ \\
sampling time point & 8 & 4895 & 4.5 & 0.064 & $<0.001$ \\
storage temperature & 2 & 476 & 1.8 & 0.006 & $<0.001$ \\
subject:sampling time point & 16 & 8923 & 4.1 & 0.117 & $<0.001$ \\
storage temperature:duration & 4 & 573 & 1.1 & 0.008 & 0.27 \\
residuals & 113 & 15237 & 0.2 & &
\end{tabular}

2

subject: three subjects $\mathrm{A}, \mathrm{B}$ and $\mathrm{C}$

sampling time point: days stools were collected

storage temperature before long-term freezing: immediately frozen, ambient, chilled, preservative at ambient duration of storage before long-term freezing: $0,2,5$ or $24 \mathrm{~h}$

7 


\section{Figure 1 (on next page)}

Overview of the stool microbial community survey and main experimental factors investigated.

Three subjects were recruited to provide stools three times over the span of three to four days (with the exception of subject $B$ at day 0,1 and 7) thrice over the duration of 72 days. Stools collected at day 0 were subjected to all four treatments to assess their influence on microbial community composition. Community profiles from stools immediately frozen at -80 ${ }^{\circ} \mathrm{C}$ were used as references for comparison. Subsequent stools collected from day 1 onwards (sampling time points indicated in figure) were immediately frozen at $-80{ }^{\circ} \mathrm{C}$ (only treatment $1)$. 


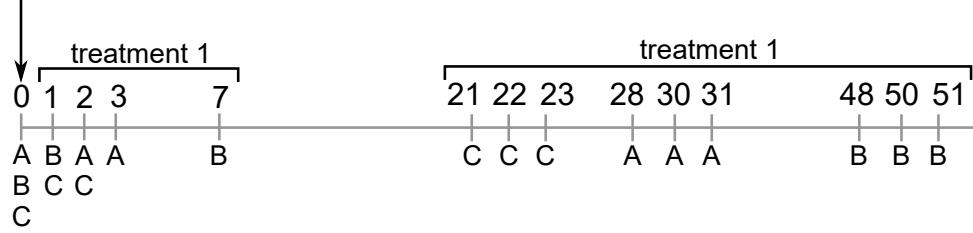

treatment 1

Sample treatments:

1. frozen immediately $\left(-80^{\circ} \mathrm{C}\right)$

2. ambient for $2,5,24 \mathrm{~h}$ then $-80^{\circ} \mathrm{C}$

3. chilled for $2,5,24 \mathrm{~h}$ then then $-80^{\circ} \mathrm{C}$

4. preservation medium, ambient for $0,2,5,24 \mathrm{~h}$ then $-80^{\circ} \mathrm{C}$ 


\section{Figure 2 (on next page)}

Principal component ordination of stool microbial community composition collected from three subjects over 72 days.

Community composition was determined by sequencing 16S rRNA gene amplicons.

Ordination of A. Samples from subject A; B. Samples from subject B; C. Samples from subject

C; D. samples from all three subjects. Each circle represents one sample, and colour of circle represents storage condition: white, immediately frozen at $-80^{\circ} \mathrm{C}$; red, ambient temperature; blue, chilled; green, preservative medium at ambient temperature. Numbers in panels A-C represent number of days subsequent samples were collected following initial sampling at day 0 (day 0 samples immediately frozen are represented by the white circles). All samples collected after day 0 were immediately frozen at $-80^{\circ} \mathrm{C}$. 
Figure 3 (on next page)

Principal component ordination of stool microbial community composition by storage condition and duration.

Community composition was determined by sequencing 165 rRNA gene amplicons. A.

Samples from subject A; B. Samples from subject B; C. Samples from subject C. Each circle represents one sample. Colour of circle represents storage condition: white, immediately frozen at $-80{ }^{\circ} \mathrm{C}$; red, ambient temperature; blue, chilled; green, preservative medium at ambient temperature. Size of circle represents duration samples were subject to their respective conditions before frozen long-term at $-80^{\circ} \mathrm{C}$. 
